\title{
Rapid Identification of Unknown Impurities in 3-Bromo-5-(trifluoromethyl)aniline by LC-SPE/NMR
}

\author{
Miroslava Harča, ${ }^{1}$ Iva Habinovec, ${ }^{2}$ Ernest Meštrović, ${ }^{1,2}$ Ivana Biljan, ${ }^{2, *}$ Predrag Novak ${ }^{2, \#}$
}

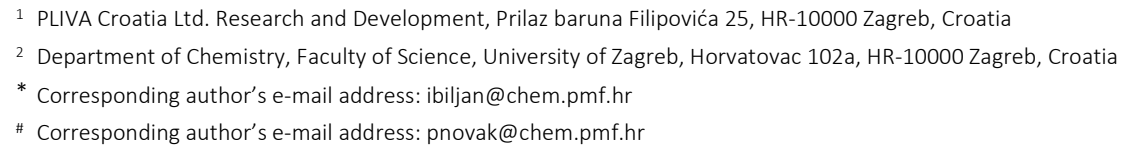

RECEIVED: November 24, 2016 * REVISED: January 17, 2017 * ACCEPTED: January 17, 2017

Abstract: Identification of unknown pharmaceutical impurities is an essential part in the drug development process. In the present study, we developed and applied liquid chromatography (LC) - solid-phase extraction (SPE)/ nuclear magnetic resonance (NMR) methodology with cryoprobe for identification and structural characterization of unknown impurities in 3-bromo-5-(trifluoromethyl)aniline. The three main impurities were separated and isolated by LC-SPE system. After multiple trapping, isolated impurities were eluted from the SPE cartridges with deuterated acetonitrile and one- and two-dimensional homo- and heteronuclear NMR spectra were recorded. The structures of the unknown impurities were determined by detailed inspection of NMR spectra and by mass spectrometric (MS) analysis. The results of the present preliminary study demonstrated that LC-SPE/NMR can be used for rapid impurity profiling of 3-bromo-5-(trifluoromethyl)aniline.

Keywords: LC-SPE/NMR, impurities, identification, structure determination.

\section{INTRODUCTION}

A $S$ impurities define the quality of a chemical entity, their unambiguous structure determination is compulsory part of the pharmaceutical development. Impurity identification provides understanding of the impurity origin and allows better control of the process. Strict regulatory guidelines (International Council for Harmonisation of technical requirements for registration of pharmaceuticals for human use - ICH, Food and Drug Administration - FDA, European Medicines Agency - EMA) made identification of low-level impurities an imperative. The determination of impurities at low levels depends on the analytical application used, indicating that no compound will be completely pure if technique sensitive enough is administered. For active pharmaceutical ingredients (APIs) with maximum daily intake less than $2 \mathrm{~g}$, regulatory agencies set the limit for unknown, unspecified impurity at $0.10 \%$ and $0.15 \%$ for known, specified impurities. ${ }^{[1-7]}$ The impurities present in API can be process impurities, degradation impurities or impurities originating from raw materials used in the synthesis. ${ }^{[8-11]}$

The structural elucidation of an unknown impurity is a complex procedure. The conventional approach to the impurity identification consists of separation of the impurity from the main component followed by isolation and multiple spectroscopic analysis and/or analysis by other standard techniques such as x-ray crystallography and mass spectrometry (MS) of the isolated impurity. Modern approach to the drug impurity profiling is based on the use of hyphenated systems, usually chromatographic with spectroscopic and/or spectrometric system. ${ }^{[12-18]}$ One of the most efficient and powerful tools for identification of compounds in complex mixtures in the pharmaceutical industry is the system comprising of liquid chromatography (LC), solid phase extraction (SPE) and nuclear magnetic resonance spectroscopy (NMR). ${ }^{[19,20]}$ The post-column peak trapping onto the SPE cartridges eliminates the need to use 
deuterated mobile phases during the chromatographic separation and enables the separated compounds to be pre-concentrated for subsequent NMR analysis. In addition, the use of cryogenically cooled probe for NMR measurements further improves the sensitivity of the method. ${ }^{[21-24]}$

3-Bromo-5-(trifluoromethyl)aniline is used as a building block in pharmaceutical industry. The trifluoro moiety can be found in several APIs such as anacetrapib, cinalcet, nilotinib, ponatinib and radotinib. ${ }^{[25-29]}$ Due to the fact that fluorine atoms can dramatically influence molecules' properties, fluorine has become unavoidable atom in drug development. ${ }^{[30]}$ In order to avoid difficult fluorination chemistry, it is usually being incorporated in the API via fluorine-containing raw material.[31]

To the best of our knowledge, no data regarding 3bromo-5-(trifluoromethyl)aniline potential impurities are available in the literature. The impact of elucidation of structures of those impurities benefits both the understanding of 3-bromo-5-(trifluoromethyl)aniline synthesis and may provide insights into the potential impurities found in the related APIs. In the present preliminary study, sample of 3-bromo-5-(trifluoromethyl)aniline was analysed by ultra high performance liquid chromatography (UHPLC) with ultraviolet diode array detection (UV-DAD) and results revealed presence of several impurities in the range of $0.1-1.5 \%$. Therefore, we developed and applied LC-SPE/NMR methodology with cryoprobe for separation, isolation and structural characterization of the three main impurities in 3-bromo5-(trifluoromethyl)aniline sample. The structures of the unknown impurities were determined by recording and interpretation of one- and two-dimensional homo- and heteronuclear NMR spectra and by MS analysis.

\section{EXPERIMENTAL}

\section{UHPLC-UV-DAD Analysis}

The UHPLC analyses were performed on an Agilent 1290 Infinity II LC system with a DAD. The UV spectra of all peaks were recorded from 190 to $400 \mathrm{~nm}$, and the working wavelength was $224 \mathrm{~nm}$. The column used was Waters Acquity UPLC BEH C18 $(100 \mathrm{~mm} \times 2.1 \mathrm{~mm}$ i.d., particle size $1.7 \mu \mathrm{m})$. The column and autosampler temperatures were $60{ }^{\circ} \mathrm{C}$ and $10^{\circ} \mathrm{C}$, respectively. Mobile phases employed for gradient elution were water/acetonitrile mixture $(95: 5, \mathrm{v} / \mathrm{v})$ as mobile phase $A$ and acetonitrile as mobile phase $B$. Gradient conditions for the analysis were: linear gradient from $40 \%$ of mobile phase B up to $60 \%$ of mobile phase $B$ from 0 to $1.5 \mathrm{~min}$; isocratic flow at $60 \%$ of mobile phase $B$ from 1.5 to $3.0 \mathrm{~min}$; linear gradient up to $95 \%$ of mobile phase B from 3.0 to $7.0 \mathrm{~min}$; linear gradient up to $40 \%$ of mobile phase B from 7.0 to $8.0 \mathrm{~min}$; and finally, reconditioning the column for $1.5 \mathrm{~min}$ on starting conditions. Typically, $2 \mu \mathrm{L}$ of methanol sample solution was injected at a flow rate of $0.4 \mathrm{~mL} \mathrm{~min}-1$.

\section{LC-SPE Analysis}

The LC-SPE analyses were performed on an Agilent 1260 Infinity HPLC system coupled with Bruker Prospekt2 SPE system comprising of make-up flow pump, high pressure dispenser, automatic cartridge exchanger module and SPE cartridge holder. The UV spectra of all peaks were recorded from 190 to $400 \mathrm{~nm}$, and the working wavelength was $224 \mathrm{~nm}$. The HPLC column used was Waters XBridge C18 ( $250 \mathrm{~mm} \times 4.6 \mathrm{~mm}$ i.d., particle size $5 \mu \mathrm{m}$ ). The column and autosampler temperatures were $50{ }^{\circ} \mathrm{C}$ and $15{ }^{\circ} \mathrm{C}$, respectively. Water (mobile phase A) and acetonitrile (mobile phase B) were used as mobile phases for gradient elution at a flow rate of $1.0 \mathrm{~mL} \mathrm{~min}^{-1}$. Gradient conditions for the analysis were: linear gradient from $32 \%$ of mobile phase $B$ up to $67 \%$ of mobile phase $B$ from 0 to $35.0 \mathrm{~min}$; reconditioning the column for $5 \mathrm{~min}$ on starting conditions. In order to increase the retention of the compounds on SPE cartridges a post-column water addition was performed with a flow rate of $3.0 \mathrm{~mL} \mathrm{~min}{ }^{-1}$. Injection volume was typically $10 \mu \mathrm{L}$. Impurities were trapped on HySphere Resin GP SPE cartridges $(10 \mathrm{~mm} \times 2 \mathrm{~mm})$.

\section{NMR Spectroscopy}

NMR spectra with NOESY-type solvent suppression module were recorded on Bruker Avance III HD 400 NMR spectrometer equipped with a broadband observed (BBO) $5 \mathrm{~mm}$ Prodigy cryoprobe and z-gradient accessories. After LC separation and analyte trapping on the SPE unit, the isolated impurities were dissolved in acetonitrile- $d_{3}$ and measured in $3 \mathrm{~mm}$ tubes with MATCH inserts for adapting the tubes to $5 \mathrm{~mm}$ Bruker spinners. All experiments were performed at $298 \mathrm{~K} .{ }^{1} \mathrm{H}$ and ${ }^{13} \mathrm{C}$ chemical shifts were referenced with respect to tetramethylsilane whereas the ${ }^{19} \mathrm{~F} \mathrm{NMR} \mathrm{spectra} \mathrm{were} \mathrm{referenced} \mathrm{to} \mathrm{CFCl}_{3}$.

${ }^{1} \mathrm{H}$ NMR spectra with spectral width of $8013 \mathrm{~Hz}$ and a digital resolution of $0.49 \mathrm{~Hz}$ per point were measured with 128 scans. ${ }^{19} \mathrm{~F}$ NMR spectra were acquired with 128 scans, the spectral width of $150 \mathrm{kHz}$ and digital resolution of $2.29 \mathrm{~Hz}$ per point. The gCOSY spectra were acquired using 2048 points in the $f 2$ dimension and 256 increments in the $f 1$ dimension. For each increment, 16 scans and the spectral width of $6394 \mathrm{~Hz}$ were applied. Digital resolution was 6.24 and $49.95 \mathrm{~Hz}$ per point in $f 2$ and $f 1$ dimensions, respectively.

The gHSQC spectra were measured with 64 scans and the spectral width of $6394 \mathrm{~Hz}$ in $f 2$ and $18112 \mathrm{~Hz}$ in $f 1$ dimension. 2048 points were used in the $f 2$ dimension and 256 increments in the $f 1$ dimension. Digital resolution was 
$6.24 \mathrm{~Hz}$ per point in $f 2$ dimension and $141.5 \mathrm{~Hz}$ per point in $f 1$ dimension. The gHMBC spectra were acquired with 128 scans and the spectral width of $5330 \mathrm{~Hz}$ in $f 2$ and $22346 \mathrm{~Hz}$ in $f 1$ dimension. 2048 points were used in the $f 2$ dimension and 256 increments in the $f 1$ dimension. The resulting digital resolution was 5.21 and $174.6 \mathrm{~Hz}$ per point in $f 2$ and f1 dimensions, respectively.

\section{UHPLC-MS Analysis}

The LC-MS analyses were performed on a Waters Acquity UPLC I-Class system with a photo diode array (PDA) detector hyphenated with Waters Acquity single quadrupole mass detector (SQD). The column used was Waters Acquity UPLC BEH C18 $(100 \mathrm{~mm} \times 2.1 \mathrm{~mm}$ i.d., particle size $1.7 \mu \mathrm{m})$. The column and autosampler temperatures were $60{ }^{\circ} \mathrm{C}$ and $10^{\circ} \mathrm{C}$, respectively. Mobile phases employed for gradient elution were water as mobile phase $\mathrm{A}$ and acetonitrile with addition of formic acid $(\varphi=$ $0.02 \%)$ as mobile phase B. Gradient conditions for the analysis were: isocratic flow at $33 \%$ of mobile phase $B$ from 0 to $2.0 \mathrm{~min}$; linear gradient up to $67 \%$ of mobile phase $B$ from 2.0 to $8.0 \mathrm{~min}$; change up to $33 \%$ of mobile phase $B$ from 8.0 to $8.1 \mathrm{~min}$; and finally, reconditioning the column for $1.0 \mathrm{~min}$ on starting conditions. Typically, $0.2 \mu \mathrm{L}$ of sample was injected at a flow rate of $0.4 \mathrm{~mL} \mathrm{~min}^{-1}$. The MS spectra were obtained under positive electrospray ionisation (ESI), capillary voltage of $1000 \mathrm{~V}$, cone voltage of $60 \mathrm{~V}$, extractor voltage of $1.00 \mathrm{~V}$, desolvation temperature of $200{ }^{\circ} \mathrm{C}$ and source temperature of $80^{\circ} \mathrm{C}$. Cone gas flow was $50 \mathrm{~L} \mathrm{~h}^{-1}$ and desolvation gas flow was $550 \mathrm{~L} \mathrm{~h}^{-1}$. Mass range in which the measurements were performed was from $\mathrm{m} / \mathrm{z} 80$ to 500 .

\section{Chemicals}

Both acetonitrile and methanol gradient grade for chromatography were purchased from J. T. Baker. Acetonitrile- $d_{3}$ was purchased from Euriso-top and formic acid (98\% p.a.) was purchased from Merck.

\section{Sample preparation}

3-Bromo-5-(trifluoromethyl)aniline sample was dissolved in methanol. Final concentrations were $0.15 \mathrm{mg} \mathrm{mL}^{-1}$ for UHPLC and $150 \mathrm{mg} \mathrm{mL}^{-1}$ for LC-SPE analysis.

\section{RESULTS AND DISCUSSION}

Prior to LC-SPE/NMR analysis, chromatographic UHPLC method for separation of impurities in 3-bromo-5(trifluoromethyl)aniline was developed and optimised with regard to the column and gradient elution. The three main unknown impurities of 3-bromo-5-(trifluoromethyl)aniline were detected by DAD during the UHPLC analysis eluting with respect to the main peak at relative retention times of

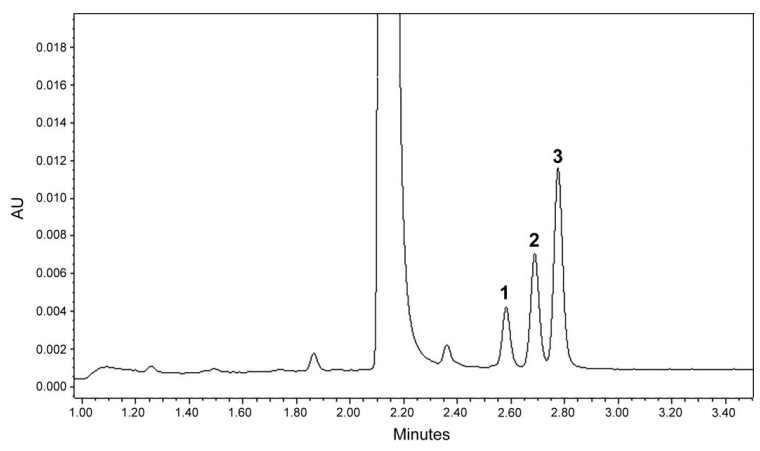

Figure 1. UHPLC-UV-DAD chromatogram of 3-bromo-5(trifluoromethyl)aniline showing impurities $1-3$ and the principal peak.

1.21 (impurity 1), 1.26 (impurity 2) and 1.31 (impurity 3) (Figure 1).

As calculated by the peak area normalization method, the sample of 3-bromo-5-(trifluoromethyl)aniline contained $0.37 \%, 0.74 \%$, and $1.33 \%$ of impurities 1,2 , and 3, respectively. Comparison of the UV spectra of 3-bromo5-(trifluoromethyl)aniline with those of impurities 1-3 suggested a structural similarity between the compounds. Retention times in LC-UV-DAD chromatogram indicated lower polarity of the impurities 1-3 when compared to the main compound. In order to elucidate the structures of the unknown impurities, compounds 1-3 were separated and isolated from the sample by LC-SPE system. The chromatographic method was transferred from UHPLC to HPLC by changing the column, adjusting the column temperature and the mobile phase flow rate. After multiple trapping of analysed impurities, performed in order to ensure a sufficient analyte quantity allowing the acquisition of NMR spectra with improved signal-to-noise ratio, the analytes were eluted from the cartridges to NMR tubes with deuterated acetonitrile. For all three impurities, one-dimensional ${ }^{1} \mathrm{H}$ and ${ }^{19} \mathrm{~F}$, and two-dimensional COSY, ${ }^{1} \mathrm{H}-{ }^{13} \mathrm{C} \mathrm{HSQC}$ and ${ }^{1} \mathrm{H}-{ }^{13} \mathrm{C} \mathrm{HMBC}$ spectra were recorded.

In the ${ }^{1} \mathrm{H}$ NMR spectrum of impurity 1 two singlets appeared in the aromatic region (at 7.71 and $7.12 \mathrm{ppm}$ ) indicating a tetrasubstituted benzene ring with two protons in para position to each other (Figure 2a). In addition, a broad singlet was observed at $5.18 \mathrm{ppm}$ and assigned to protons of the amino group. The ${ }^{19} \mathrm{~F}$ NMR spectrum displayed a signal at $-61.07 \mathrm{ppm}$ attributed to the trifluoromethyl group. The analysis of the ${ }^{1} \mathrm{H}$ NMR spectrum of impurity 2 revealed two doublets in the aromatic region (at 7.18 and $7.15 \mathrm{ppm}$ ) corresponding to two protons (Figure $2 \mathrm{~b}$ ). The value of coupling constant between these two protons was measured to be $2.1 \mathrm{~Hz}$. This indicated that impurity $\mathbf{2}$ contains four substituents on benzene ring with two protons in meta position to each 
other. A broad singlet at $5.07 \mathrm{ppm}$ which can be attributed to protons of the amino group also appeared in the ${ }^{1} \mathrm{H} N M R$ spectrum. The ${ }^{19} \mathrm{~F}$ NMR spectrum revealed a signal at $-63.2 \mathrm{ppm}$ assigned to the trifluoromethyl group. The ${ }^{1} \mathrm{H}$ NMR spectrum of impurity 3 showed two multiplets in the aromatic region (at 7.22-7.21 and 7.03-7.02 ppm) corresponding to two protons and indicating that impurity $\mathbf{3}$ also contains four substituents on benzene ring (Figure 2c). Furthermore, the broad singlet at 5.15 ppm was observed in the ${ }^{1} \mathrm{H}$ NMR spectrum and attributed to the protons of the amino group whereas the ${ }^{19} \mathrm{~F}$ NMR spectrum showed a signal at $-63.76 \mathrm{ppm}$ which can be assigned to the trifluoromethyl group. The correlation peaks in 2D COSY spectrum of impurity 3 suggested that the two aromatic protons are in meta position to each other. Analysis of multiplets in the ${ }^{1} \mathrm{H}$ NMR spectrum of impurity 3 indicated that the signals of the aromatic protons are split into doublets because of meta coupling between them, and doublets are further split into quartets due to coupling with fluorine atoms of the trifluoromethyl group (the inset in Figure 2c). The splitting pattern of aromatic protons in ${ }^{1} \mathrm{H}$ NMR spectrum of impurity 3 thus confirmed that the two protons are adjacent to the trifluoromethyl group. The analysis of HSQC spectra of impurities 1-3 enabled unambiguous assignment of the protonated carbon atoms while the inspection of $\mathrm{HMBC}$ spectra revealed information about the quaternary carbon atoms. The presented NMR data suggested that impurities $\mathbf{1 - 3}$ are tetrasubstituted benzene derivatives containing amino and triflouromethyl groups and the two additional substituents.

In order to deduce the complete structures, the solutions of isolated impurities 1-3 were further analysed by LC coupled with a single quadrupole mass spectrometer. As

a)

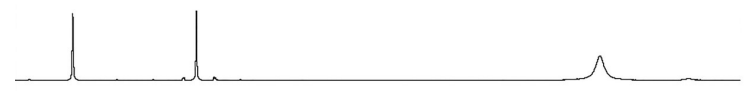

b)

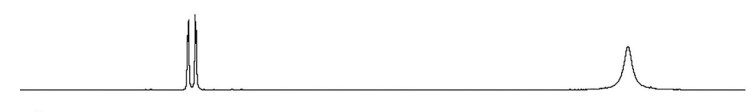

c)

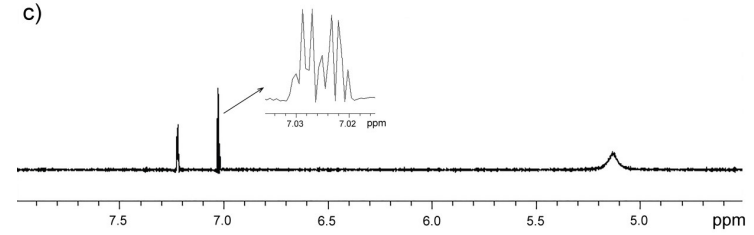

Figure 2. ${ }^{1} \mathrm{H}$ NMR spectra of impurities (a) 1 ; (b) 2; and (c) 3 in acetonitrile- $d_{3}$ recorded in the LC-SPE/NMR mode. The inset in c) shows quartet of doublets splitting pattern of aromatic protons in ${ }^{1} \mathrm{H}$ NMR spectrum of impurity 3 . mobile phases used for LC-UV-DAD analysis of 3-bromo-5(trifluoromethyl)aniline contained only water and acetonitrile, it was necessary to modify them in order to ensure the ionisation of the compounds. Therefore, formic acid was used as an additive. LC-MS chromatograms were comparable to the chromatograms obtained in the initial testing of the 3-bromo-5-(trifluoromethyl)aniline sample. Peaks were tracked by comparing the retention times and UV spectra of separated components. ESI-MS spectra acquired with a UHPLC-UV-DAD-MS hyphenated system revealed that all three isolated impurities are isomers. The difference from the elemental composition of 3-bromo-5(trifluoromethyl)aniline compound was an additional bromine atom. This was confirmed by isotope distribution characteristic for molecules with two bromine atoms (1:2:1) (Figure 3). The fragmentation that is most likely to occur is the observed elimination of one bromine atom $(\mathrm{m} / \mathrm{z}$ 318:320:322 $\rightarrow m / z$ 238:240).

The results of LC-MS analysis along with the assignment of NMR resonances and inspection of the correlation peaks in HMBC spectra (Figure 4) providing information on the relative position of the substituents on the benzene rings allowed for structural identification of

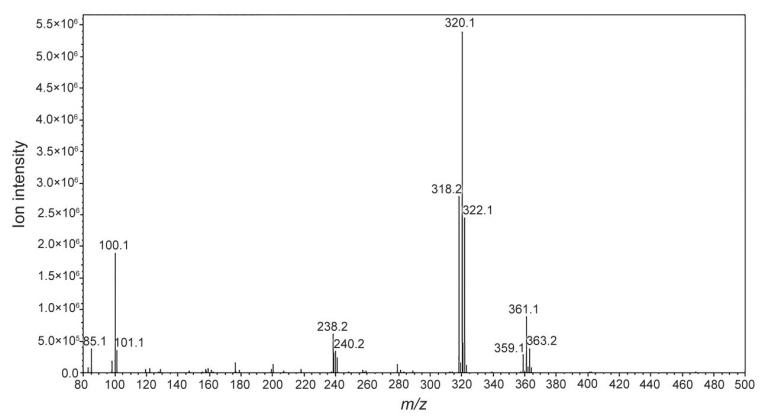

Figure 3. ESI-MS spectrum of impurity 3.

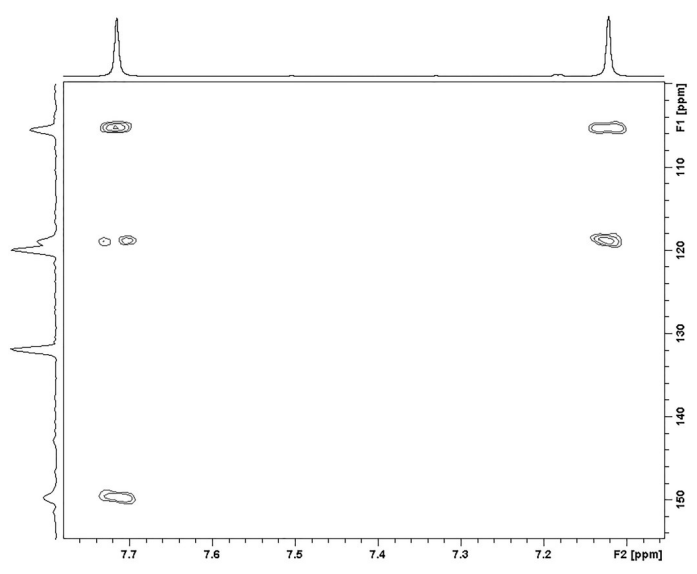

Figure 4. HMBC spectrum of impurity 1 obtained in the LC$\mathrm{SPE} / \mathrm{NMR}$ mode showing long range ${ }^{1} \mathrm{H}-{ }^{13} \mathrm{C}$ correlation peaks. 
<smiles>Nc1cc(C(F)(F)F)c(Br)cc1Br</smiles>

Impurity 1<smiles>Nc1cc(Br)cc(C(F)(F)F)c1Br</smiles>

Impurity 2

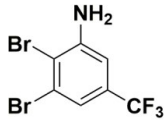

Impurity 3
Scheme 1. Proposed structures of main impurities $1-3$ in 3-bromo-5-(trifluoromethyl)aniline sample.

impurities 1-3. The proposed structures of the three main impurities in 3-bromo-5-(trifluoromethyl)aniline are shown in Scheme 1.

In conclusion, this preliminary study has demonstrated that LC-SPE/NMR system with cryoprobe can be used successfully for rapid isolation and identification of unknown impurities in 3-bromo-5(trifluoromethyl)aniline. After the three main impurities were separated by LC and trapped on SPE cartridges, oneand two-dimensional NMR spectra were recorded. The analysis of NMR spectra and results of MS measurements suggested that the three main impurities present in 3bromo-5-(trifluoromethyl)aniline are di-bromo derivatives of 3-(trifluoromethyl)aniline. Studies regarding the identification of additional impurities in 3-bromo-5(trifluoromethyl)aniline by using LC-SPE/NMR approach are in progress in our laboratory.

Acknowledgment. This work was supported by the European Regional Development Fund and Croatian state budget (project Met4Pharm).

\section{REFERENCES}

[1] R. Holm, D. Elder, Eur. J. Pharm. Sci. 2016, 87, 118.

[2] ICH Harmonized Tripartite Guideline, Q3A(R2): Impurities in New Drug Substances, 2006.

[3] ICH Harmonized Tripartite Guideline, Q3B(R2): Impurities in New Drug Products, 2006.

[4] EMA, ICH guidelines, can be found under http://www.ema.europa.eu/ema/index.jsp?curl=pag es/regulation/general/general_content_000 035.jsp.

[5] FDA, ICH Guidance Documents, can be found under http://www.fda.gov/ScienceResearch/SpecialTopic s/RunningClinicalTrials/GuidancesInformationSheet sandNotices/ucm219488.htm.

[6] N. Mistry, I. M. Ismail, M. S. Smith, J. K. Nicholson, J. C. Lindon, J. Pharm. Biomed. Anal. 1997, 16, 697.

[7] S. Thomas, C. S. Mathela, A. Agarwal, S. K. Paul, J. Pharm. Biomed. Anal. 2011, 56, 423.

[8] F. Qiu, D. L. Norwood, J. Liq. Chromatogr. Relat. Technol. 2007, 30, 877.

[9] S. Görög, Anal. Bioanal. Chem. 2003, 377, 852.

[10] D. Bartos, S. Görög, Curr. Pharm. Anal. 2008, 4, 215.
[11] S. Vasanti, S. Sulabha, Drug Inv. Today 2009, 1, 81.

[12] S. Provera, L. Rovatti, L. Turco, S. Mozzo, A. Spezzaferri, S. Bacchi, A. Ribecai, S. Guelfi, A. Mingardi, C. Marchioro, D. Papini, J. Pharm. Biomed. Anal. 2010, 53, 517525.

[13] S. Singh, T. Handa, M. Narayanam, A. Sahu, M. Junwal, R. P. Shah, J. Pharm. Biomed. Anal. 2012, 69, 148.

[14] N. Ferreirós, S. Dresen, R. M. Alonso, W. Weinmann, J. Chromatogr. B 2007, 855, 134.

[15] P. Novak, P. Tepeš, M. Cindrić, M. Ilijaš, S. Dragojević, K. Mihaljević, J. Chromatogr. A 2004, 1033, 299.

[16] P. Novak, P. Tepeš, I. Fistrić, I. Bratoš, V. Gabelica, J. Pharm. Biomed. Anal. 2006, 40, 1268.

[17] P. Novak, P. Tepeš, M. Ilijaš, I. Fistrić, I. Bratoš, A. Avdagić, V. Gabelica Marković, M. Dumić, J. Pharm. Biomed. Anal. 2009, 50, 68.

[18] P. Novak, M. Cindrić, P. Tepeš, S. Dragojević, M. Ilijaš, K. Mihaljević, J. Sep. Sci. 2005, 28, 1442.

[19] H. Kovacs, D. Moskau, M. Spraul, Prog. Nucl. Magn. Reson. Spectrosc. 2005, 46, 131.

[20] R. Mukhopadhyay, Anal. Chem. 2007, 79, 7959.

[21] C. Pan, F. Liu, Q. Ji, W. Wang, D. Drinkwater, R. Vivilecchia, J. Pharm. Biomed. Anal. 2006, 40, 581.

[22] F. Rinaldi, J. Fan, C. Pathirana, V. Palaniswamy, Magn. Reson. Chem. 2013, 51, 517522.

[23] C. Pan, F. Liu, M. Motto, J. Pharm. Sci. 2011, 100, 1228.

[24] I. Habinovec, T. Jednačak, P. Novak, ADMET \& DMPK 2015, 3, 352.

[25] M. Barniol-Xicotaa, R. Leivaa, C. Escolanob, S. Vázquez, Synthesis 2016, 48, 783.

[26] E. Y. Tan, G. Hartmann, Q. Chen, A. Pereira, S. Bradley, G. Doss, A. Shiqiang Zhang, J. Z. Ho, M. P. Braun, D. C. Dean, W. Tang, S. Kumar, Drug Metab. Dispos. 2010, 38, 459.

[27] B. J. Deadman, M. D. Hopkin, I. R. Baxendaleb, S. V. Ley, Org. Biomol. Chem. 2013, 11, 1766.

[28] W.-S. Huang, C. A. Metcalf, R. Sundaramoorthi, Y. Wang, D. Zou, R. M. Thomas, X. Zhu, L. Cai, D. Wen, S. Liu, J. Romero, J. Qi, I. Chen, G. Banda, S. P. Lentini, S. Das, Q. Xu, J. Keats, F. Wang, S. Wardwell, Y. Ning, J. T. Snodgrass, M. I. Broudy, K. Russian, T. Zhou, L. Commodore, N. I. Narasimhan, Q. K. Mohemmad, J. Iuliucci, V. M. Rivera, D. C. Dalgarno, T. K. Sawyer, T. Clackson, W. C. Shakespeare, J. Med. Chem. 2010, 53, 4701.

[29] S.-K. Heo, E.-K. Noh, D.-J. Yoon, J.-C. Jo, Y. Choi, S. J. Koh, J. H. Baek, J.-H. Park, Y. J. Min, H. Kim, PLoS One 2015, 10, e0129853.

[30] S. Swallow, Prog. Med. Chem. 2015, 54, 65.

[31] H.-J. Federsel, Bioorg. Med. Chem. 2010, 18, 5775. 